\title{
Single-patch, 2-patch, and caval division techniques for repair of partial anomalous pulmonary venous connections: Does it matter?
}

\author{
Sameh M. Said, MD, ${ }^{\text {a }}$ Harold M. Burkhart, MD,${ }^{\text {a }}$ Hartzell V. Schaff, MD, ${ }^{\text {a }}$ Frank Cetta, Jr, MD, ${ }^{b}$ \\ Sabrina D. Phillips, MD, ${ }^{\mathrm{b}}$ Roxann D. Barnes, MD, ${ }^{\mathrm{c}}$ Zhuo Li, MS, ${ }^{\mathrm{d}}$ and Joseph A. Dearani, MD ${ }^{\mathrm{a}}$
}

Objective: We reviewed our experience with the single-patch, 2-patch, and caval division techniques for repair of a partial anomalous pulmonary venous connection.

\begin{abstract}
Methods: From 1990 to 2009, 124 patients (65 male patients; 52\%) were identified. The single-patch, twopatch, and caval division technique was used in $60(49 \%), 24(19 \%)$, and $40(32 \%)$ patients, respectively. The median age was 34.5 years (range, 1-76). Of the 124 patients, $99(80 \%)$ had an atrial septal defect.

Results: There was no early mortality. Survival at 5, 10, and 15 years was $99 \%, 95 \%$, and $82 \%$, respectively. One patient $(3 \%)$ in the caval division group required early reoperation for superior vena caval obstruction. All patients, exept for 3 , had regained sinus rhythm at discharge. Late superior vena caval obstruction developed in 3 patients $(7.5 \%)$ in the caval division group, $3(5 \%)$ in the single-patch group, and $1(4 \%)$ in the 2-patch group $(\mathrm{P}=.5)$. Two patients underwent reoperation for late pulmonary vein obstruction: $1(2 \%)$ in the single-patch group and $1(4 \%)$ in the 2-patch group $(P=.08)$. A permanent pacemaker was required in 3 patients $(3 \%)$.

Conclusions: Surgical treatment of partial anomalous pulmonary venous connections is associated with excellent outcomes. The overall incidence of late superior vena caval or pulmonary vein stenosis is low. Although not significant, the 2-patch technique might be associated with a greater incidence of sinus node dysfunction and late pulmonary venous stenosis. The late development of superior vena caval obstruction is a concern with all techniques, necessitating close follow-up. (J Thorac Cardiovasc Surg 2012;143:896-903)
\end{abstract}

Partial anomalous pulmonary venous connection (PAPVC) includes those cardiovascular anomalies in which 1 or more, but not all, of the pulmonary veins connect to the right atrium directly or indirectly by way of different systemic venous connections. It occurs in about $0.6 \%$ to $0.7 \%$ of the population, ${ }^{1}$ according to autopsy data, but the actual incidence could be greater, ${ }^{2}$ because the finding of such an anomaly is not uncommon. PAPVC can occur as an isolated anomaly, although it is commonly associated with a sinus venosus type of atrial septal defect (ASD).

The main principle for anatomic repair of such anomalies usually requires the creation of an intra-atrial baffle to redirect the anomalous pulmonary venous drainage to the left atrium. A number of corrective procedures have been described, including single-patch, 2-patch, and caval division procedures. Surgical correction of such anomalous drainage has excellent outcomes overall. The main concern with

From the Division of Cardiovascular Surgery, ${ }^{\mathrm{a}}$ Division of Pediatric Cardiology, Division of Cardiac Anesthesia, ${ }^{c}$ and Division of Biomedical Statistics and Informatics, ${ }^{\mathrm{d}}$ Mayo Clinic, Rochester, Minn.

Disclosures: Authors have nothing to disclose with regard to commercial support.

Read at the 37th Annual Meeting of the Western Thoracic Surgical Association, Colorado Springs, Colorado, June 22-25, 2011.

Received for publication June 21, 2011; revisions received Aug 14, 2011; accepted for publication Sept 26, 2011; available ahead of print Feb 9, 2012.

Address for reprints: Harold M. Burkhart, MD, Division of Cardiovascular Surgery, Mayo Clinic, 200 First Street Southwest, Rochester, MN 55905 (E-mail: burkhart. harold@mayo.edu).

$0022-5223 / \$ 36.00$

Copyright (C) 2012 by The American Association for Thoracic Surgery doi:10.1016/j.jtcvs.2011.09.074 these techniques has been related to the risk of superior vena cava (SVC) or pulmonary venous obstruction and the occurrence of postoperative dysrhythmias or sinus node dysfunction. ${ }^{3}$ We analyzed our results with these different surgical techniques for repair of PAPVC.

\section{METHODS \\ Patient Population}

From February 1990 to August 2009, 178 patients with PAPVC were identified. Those with isolated left-sided PAPVC and those who had undergone previous PAPVC surgery were excluded from the present cohort. A total of 124 patients were identified who were undergoing surgical repair for right-sided PAPVC. Three different surgical techniques were used: the single-patch technique in 60 patients $(49 \%)$, the 2-patch technique in 24 patients $(19 \%)$, and caval division in 40 patients $(32 \%)$. The mean age at repair was $34.9 \pm 23$ years (range, 1-76). Of the 124 patients, 65 were males $(52 \%)$ and 59 were females $(48 \%) ; 36(29 \%)$ were children. Using the caval division technique, 18 patients (45\%) underwent the traditional Warden procedure, 17 (43\%) underwent a modified version of Warden using a short interposition graft to lengthen the SVC, and 5 patients $(12 \%)$ underwent caval division without reimplantation. The Mayo Clinic and Foundation institutional review board approved the present retrospective review.

\section{Preoperative Data}

The presence of symptoms or signs of right ventricular volume overload was the main indication for surgery. Of the 124 patients, $50(40 \%)$ were asymptomatic. The most common symptoms were exertional dyspnea in 61 patients $(49 \%)$ and fatigue in $25(20 \%)$. Preoperative arrhythmias were present in 16 patients $(13 \%)$, with atrial fibrillation in $14(11 \%)$ and atrial flutter in $2(2 \%)$. PAPVC was not identified during previous cardiac surgeries in 10 patients $(8 \%)$. Preoperative echocardiographic data 


\section{Abbreviations and Acronyms \\ ASD $=$ atrial septal defect \\ PAPVC $=$ partial anomalous pulmonary venous connection \\ RAA = right atrial appendage \\ SVC = superior vena cava}

were obtained for all patients. An ASD was found in 99 patients $(80 \%)$, with 36 patients $(29 \%)$ having more than 1 type of ASD. Sinus venosus was the most common ASD, found in 78 patients $(63 \%)$, with a patent foramen ovale and ostium secundum ASD present in $38(31 \%)$ and $18(14 \%)$ patients, respectively. Anomalous drainage of the right upper and middle lobe veins was the most common PAPVC combination in 78 patients $(61 \%)$. The mean number of the anomalous veins was 2.1 (range, $1-4$ ). Persistent left SVC was present in 16 patients $(13 \%)$, with 5 of these patients having well-developed bilateral SVC with a well-developed communicating vein. Associated left PAPVC was present in 5 patients (4\%).

\section{Surgical Technique}

Standard median sternotomy was the most common approach in 118 patients $(95 \%)$, and minimally invasive approaches were used in 2 patients ( $2 \%$; lower partial sternotomy in 1 and robotic-assisted in 1$)$. Right thoracotomy was used in the remaining 4 patients $(3 \%)$. In general, the pericardium was opened, and the heart was exposed. The SVC was dissected out to the innominate vein, and the azygous and anomalous pulmonary veins were adequately exposed. Cardiopulmonary bypass was established using standard ascending aortic and bicaval cannulation. The SVC cannula was placed as high as possible in the SVC or in the innominate vein. A persistent left SVC was managed by an additional suction line inserted into the coronary sinus through the right atrium. The mean cardiopulmonary bypass time was $78 \pm 39$ minutes, with a mean aortic crossclamp time of $40 \pm$ 23 minutes. Normothermia was used in 72 patients $(58 \%)$. A surgical ASD was created in those patients who had an intact atrial septum by excising the fossa ovalis and extending the incision upward, with removal of a portion of the septum secundum as needed to ensure an adequately size ASD with an unobstructed pathway for the anomalous pulmonary veins.

In cases of associated secundum type ASD, we typically extended the ASD superiorly, as described in the previous paragraph. The anomalous pulmonary veins were then baffled with an internal patch through the ASD into the left atrium. In the single-patch technique, the right atrium was closed primarily, and in the 2-patch technique, an autologous pericardial patch was used for closure of the SVC-right atrium junction (Figure 1). The most commonly used intra-atrial patch materials were untreated autologous pericardium in 65 patients $(52 \%)$ and bovine pericardium in 43 patients $(35 \%)$; glutaraldehyde-treated autologous pericardium, polytetrafluoroethylene (Gore-Tex; W. L. Gore \& Associates, Flagstaff, Ariz) and Dacron patches were used in $11(9 \%), 4(3 \%)$, and $1(1 \%)$ patient, respectively. To avoid isolating the sinus node from the rest of the right atrial wall during patch suturing and to minimize the risk of sinus node dysfunction, we believe that the depth of the suture bites should be partial thickness. These basic surgical principles did not differ among the 3 groups.

For those patients who underwent caval division, the azygous vein was divided and the right SVC was crossclamped and divided above the insertion of the highest anomalous pulmonary vein with oversewing of its caudal end. In 18 patients in the caval division group, the proximal portion of the SVC was anastomosed directly to the right atrial appendage (RAA) after cutting all the trabeculations, and in 17, a short segment of a ringed Gore-Tex graft was used as an interposition graft between the proximal SVC and the RAA (Figure 2). The graft sizes ranged from 14 to $20 \mathrm{~mm}$.
In the remaining 5 patients with bilateral well-developed SVC and a communicating vein, we did not reimplant the right SVC into the RAA (Figure 3).

The most common associated procedures were the modified Cox-Maze procedure using cryoablation or radiofrequency in 17 patients (14\%), tricuspid valve annuloplasty in $16(13 \%)$, ligation of the left atrial appendage in $7(6 \%)$, ventricular septal defect closure in $4(3 \%)$, and pulmonary valve replacement in $3(2 \%)$.

\section{Follow-up}

The mean follow-up period from surgery to the last examination was 6 \pm 5.8 years, maximum of 20 years. This was shorter in the modified Warden group. This difference resulted from the recent application of this technique at our institution (2006). Follow-up echocardiographic data and electrocardiograms were available for 123 patients $(99 \%)$. The electrocardiogram was considered early if it was obtained before hospital discharge and late if obtained after discharge. The electrocardiogram was interpreted as normal sinus rhythm, junctional rhythm, and supraventricular arrhythmias, which included atrial flutter, fibrillation, and multifocal atrial tachycardias.

\section{Statistical Analysis}

Categorical variables are reported as the number and percentages. Continuous variables are expressed as the mean \pm SD or the median and interquartile range. The change in continuous variables was tested using a paired $t$ test or signed rank test. The Kaplan-Meier method was used to estimate survival rate at 1,5 , and 10 years. Log-rank test was used to compare survival among the groups.

\section{RESULTS}

There was no early mortality. Early reoperation was required in 3 adult patients $(2 \%)$. The first patient was a 62-year-old man who underwent a classic Warden procedure that was complicated by SVC obstruction in the early postoperative period despite adequate SVC and RAA mobilization and a tension-free anastomosis. He required reoperation on the same day with conversion to a modified Warden with insertion of a 16-mm ringed Gore-Tex interposition graft. One patient in the single-patch group underwent reoperation for postoperative bleeding. Another patient in the Warden group underwent sternal debridement and rewiring because of sternal dehiscence.

A change in the normal sinus rhythm to a junctional rhythm was noted in 5 patients $(21 \%)$ in the 2-patch group, 8 patients $(20 \%)$ in the caval division group, and 7 patients $(12 \%)$ in the single-patch group. This had recovered at discharge, with all but 3 patients remaining in sinus rhythm at late follow-up. Supraventricular arrhythmias (Figure 4, A) were noted in 9 patients $(22 \%)$ in the caval division group versus 3 patients $(12 \%)$ in the 2-patch group and 5 patients $(8 \%)$ in the single-patch group. Sinus node dysfunction (Figure 4, B) occurred in 3 patients: 2 patients in the 2-patch group ( $8 \%$ ) and 1 patient in the caval division group $(2 \%)$. No patient in the single-patch group had sinus node dysfunction. Three patients required permanent pacemaker placement, 2 patients ( 1 in the caval division group and 1 in the 2-patch group) had preoperative atrial fibrillation and underwent concomitant modified Cox maze cryoablation 

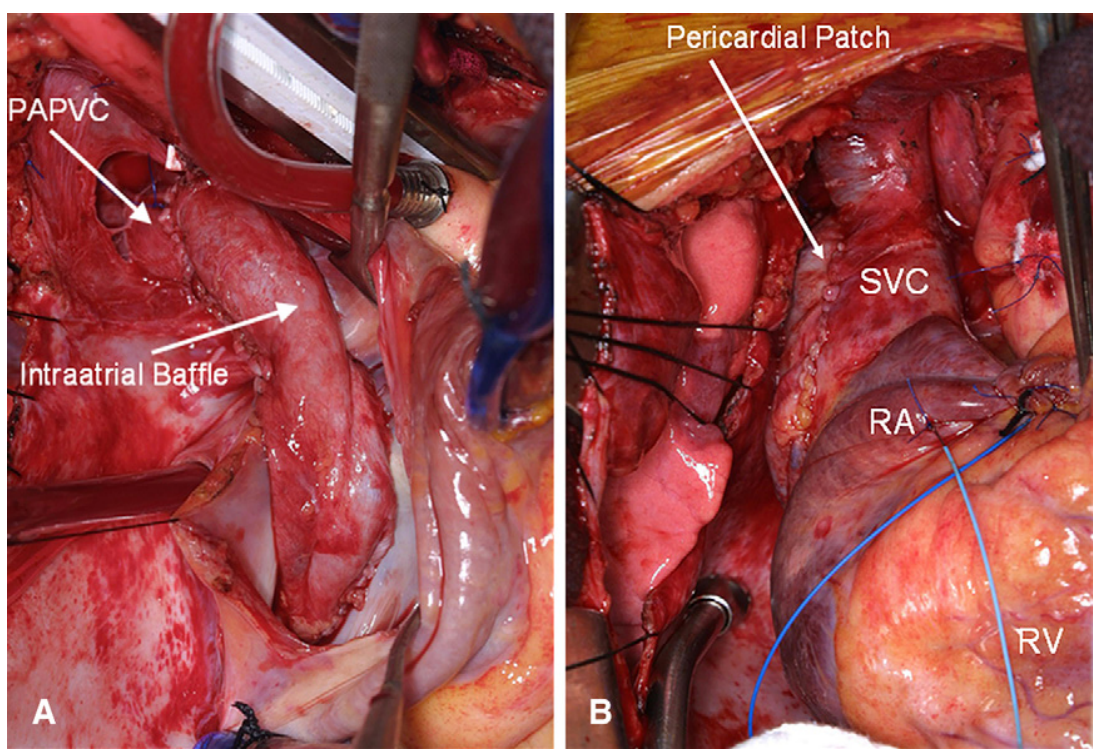

FIGURE 1. Intraoperative photographs demonstrating 2-patch technique. A, Creation of intra-atrial baffle using autologous pericardium, and B, another patch used to close the cavoatrial junction. RA, Right atrium; SVC, superior vena cava.

but developed tachy-brady syndrome postoperatively. The third patient (2-patch group) had dilated cardiomyopathy with recurrent syncopal episodes. Long-term survival was excellent among all 3 groups, with a 5-, 10-, and 15-year survival of $99 \%, 95 \%$, and $82 \%$, respectively, and with no significant difference among the 3 groups $(P=.99)$. Late mortality occurred only in adults in their seventh or eighth decades, and included 7 patients $(6 \%)$. The cause of death was noncardiac related in 5 patients, and the other 2 patients died with an advanced degree of congestive heart failure and restrictive cardiomyopathy.

Late pulmonary venous obstruction developed in 6 patients: 3 in the 2-patch group $(12 \%)$ and $3(5 \%)$ in the single-patch group (Figure 4,C). One patient, a 74-yearold woman, underwent a 2-patch technique and developed a severe right superior pulmonary vein stenosis 3 years later that required balloon angioplasty. Only 2 patients required reoperation for intra-atrial baffle obstruction. The first patient was a 10-year-old boy who developed severe right lower lobe pulmonary vein stenosis that required reoperation 14 months after his original procedure. His original intra-atrial baffle (autologous pericardium) was augmented with a ringed Gore-Tex patch. However, his pulmonary venous obstruction recurred 1 year later, and he underwent a hybrid procedure with placement of a pulmonary vein stent. The second patient was a 64-year old man who underwent a 2-patch technique for repair of PAPVC. He underwent reoperation 2 years later for relief of the intra-atrial baffle obstruction. The original autologous pericardial patch was augmented with a Gore-Tex patch. Baffle obstruction recurred again, 5 years later, and required another reoperation with patch augmentation of the baffle, in addition to tricuspid valve replacement. No patient required late reoperation in the caval division group for pulmonary venous obstruction.

Three patients $(7 \%)$ in the traditional Warden group had late SVC obstruction. The first 2 patients were 2-year-old girls who were diagnosed with SVC obstruction 10 and 8 months postoperatively, and the third was a 47 -year-old woman who was diagnosed 20 months postoperatively. No patient developed SVC obstruction after the modified Warden procedure. All late SVC obstructions were managed with percutaneous balloon angioplasty and stent placement, with no residual obstruction. Late SVC stenosis as determined by increased echocardiographic Doppler velocities was identified in 3 patients $(5 \%)$ in the single-patch group and in 1 patient (4\%) in the 2-patch group (Figure 4, $D$ ). These did not require any form of intervention.

\section{DISCUSSION}

Surgical repair of PAPVC using the single-patch, 2-patch, or caval division techniques has excellent outcomes overall. The risks associated with these procedures include postoperative dysrhythmias, sinus node dysfunction, and SVC or pulmonary vein obstruction. ${ }^{4,5}$

The idea behind the 2-patch technique is to minimize the incidence of SVC obstruction ${ }^{6}$ secondary to the pulmonary venous baffle pathway. Iyer and colleagues ${ }^{7}$ reported a total of 37 patients who underwent repair of PAPVC. Eighteen patients underwent single-patch repair, while the twopatch technique was applied in the remaining 19 patients. The mean follow-up in this series was 22 months. Significant SVC obstruction was identified in six patients in the single-patch group and in two patients in the two-patch group. No cardiac catheterization was preformed in any of these patients. In our series, the follow-up was longer 

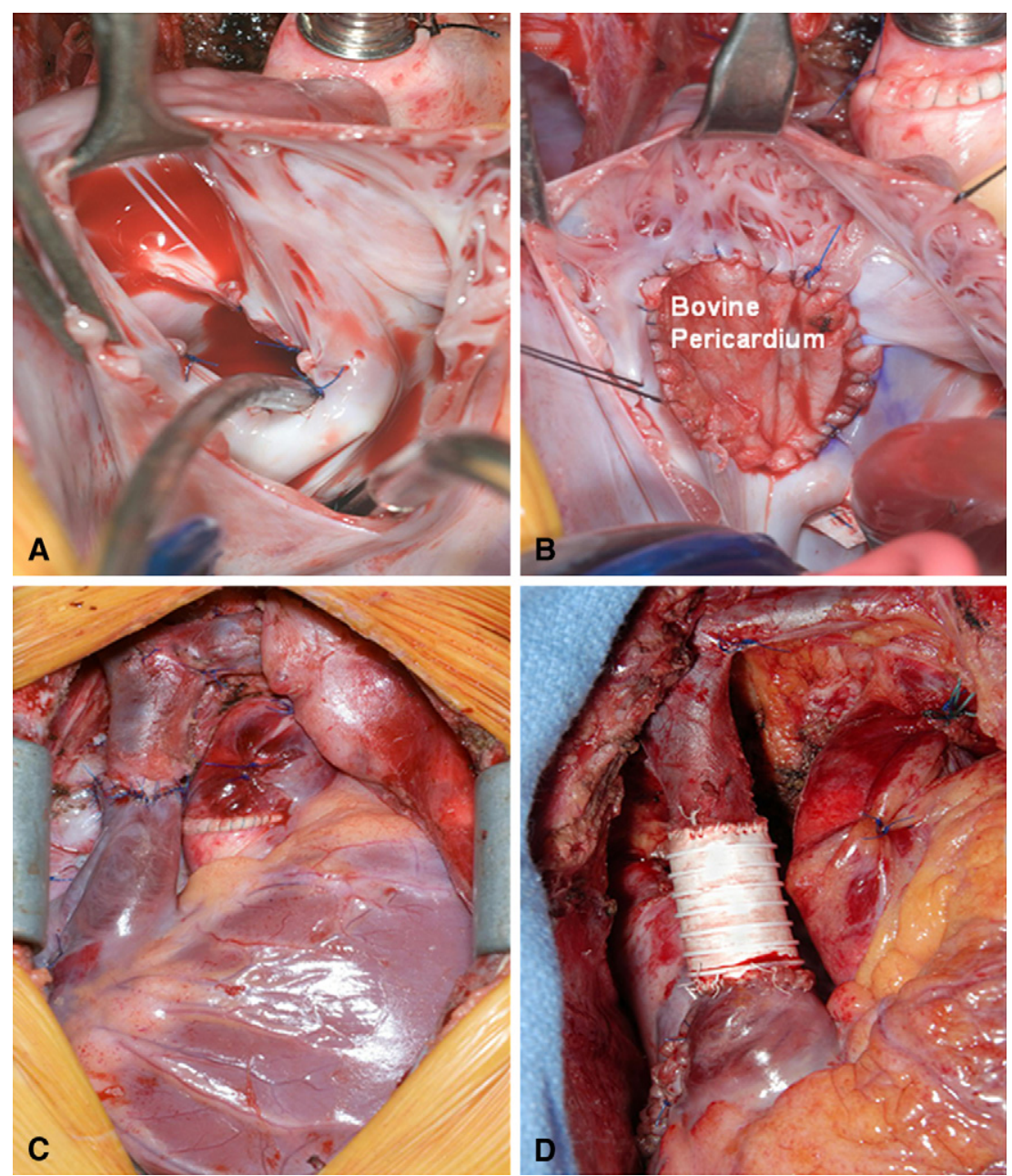

FIGURE 2. A, Surgically created atrial septal defect shown through the right atriotomy; B, bovine pericardial patch used as an intra-atrial baffle to redirect the pulmonary venous drainage through the atrial septal defect into the left atrium; $\mathrm{C}$, traditional Warden procedure with direct anastomosis between the proximal superior vena cava and the right atrial appendage; and D, modified Warden procedure using a short-ring Gore-Tex graft interposition.

with a mean of $6 \pm 5.8$ years and up to 20 years, and no patient had early SVC obstruction among our single- or two-patch techniques. There was no statistically significant difference in the late occurrence of SVC obstruction between the single-patch and the 2-patch techniques $(5 \%$ vs $4 \%$ ), and it did not require any interventions.

In managing high insertion of anomalous pulmonary veins into the SVC above the cavoatrial junction, we have adopted the caval division procedure. Gustafson and colleagues ${ }^{8}$ reported 1 case of SVC obstruction among their series of 40 patients who underwent the Warden procedure. Stewart and colleagues ${ }^{9}$ identified 1 case of SVC obstruction among a series of 5 patients, but they attributed that to inadequate excision of all the RAA trabeculations, as recommended in earlier reports. ${ }^{10}$ In our series of 18 traditional Warden procedures, we had 1 early and 3 late cases of SVC obstruction. This occurred despite adequate mobilization of the SVC and RAA and creation of a tension-free anastomosis. This was the main factor behind modifying the technique with the addition of an interposition graft for SVC reconstruction. We did not identify any case of SVC obstruction among the 17 patients who underwent the modified Warden procedure.

The occurrence of intra-atrial baffle obstruction and the late development of pulmonary venous obstruction is a real concern with surgical repair of PAPVC. Among the 37 patients reported by Iyer and colleagues, ${ }^{7}$ pulmonary venous obstruction was found in 9 patients in the single-patch group, but no patient in the 2-patch group had any gradient across the pulmonary veins. We agree with the investigators that the size of the single patch is important, with smaller patch sizes increasing the risk of pulmonary venous obstruction, although a larger patch can lead to SVC obstruction. In our series, we did not identify any significant difference between the single- and 2-patch techniques in the late development of pulmonary venous obstruction. One patient in each group underwent reoperation for relief of baffle obstruction. Both patients' original intra-atrial 


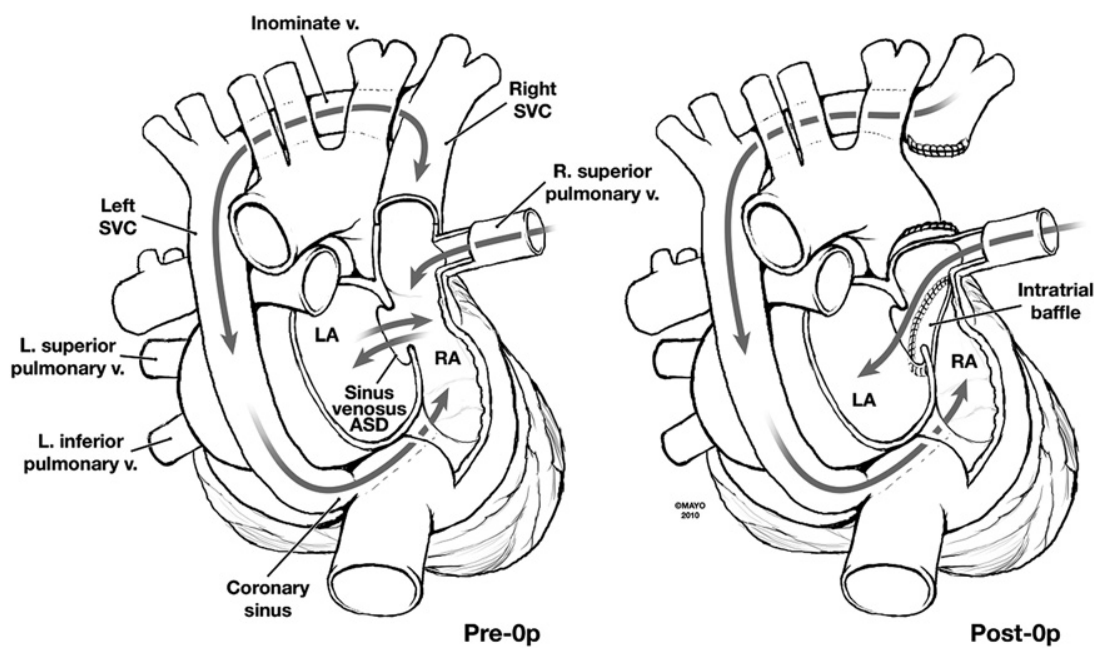

FIGURE 3. Posterior view of the heart in the 5 cases with bilateral superior vena cavae with a well-developed communicating vein. These patients underwent right superior vena cava division without reimplantation. The systemic venous drainage was directed through the communicating vein into the left superior vena cava and then through the coronary sinus into the right atrium.

baffles were constructed of untreated autologous pericardium, raising the same concern shared by others, ${ }^{11}$ regarding autologous pericardial patch contraction in the long term. Shahriari and colleagues ${ }^{11}$ switched to the Gore-Tex patch after the development of pulmonary venous obstruction in 1 patient among their series of 13 patients who underwent the Warden procedure. Among our caval division series of 40 patients, we did not identify any case of
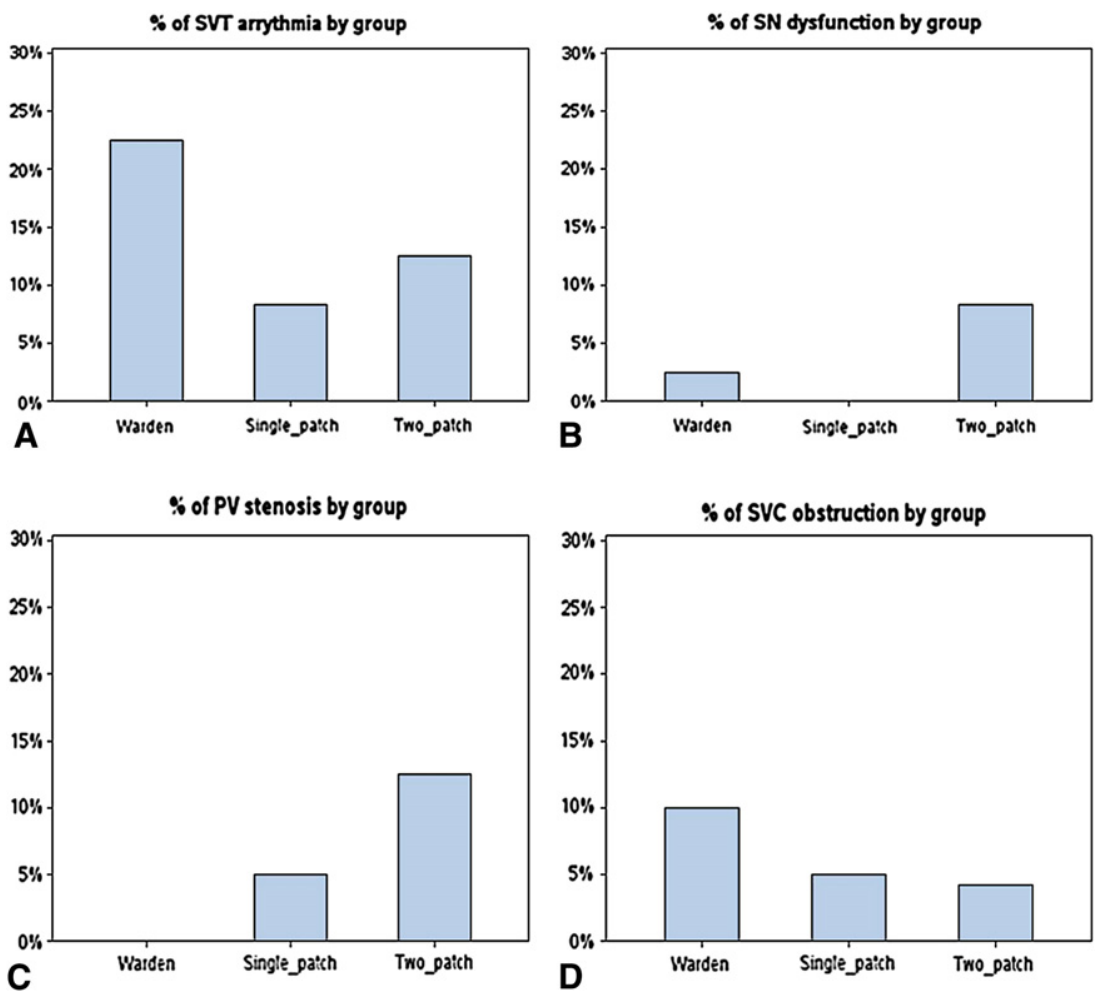

FIGURE 4. A, Graph showing the incidence of supraventricular arrhythmias in our series, identified in 9 patients $(22 \%)$ in the caval division group, 3 $(12 \%)$ in the 2-patch group, and $5(8 \%)$ in the single-patch group. B, Graph showing the incidence of sinus node dysfunction in our series, identified in 3 patients-2 patients in the 2-patch group $(8 \%)$ and 1 patient in the caval division group (2\%). No patient in the single-patch group had sinus node dysfunction. C, The incidence of late pulmonary venous obstruction, which occurred in 6 patients in our series: 3 in the 2 -patch group (12\%) and $3(5 \%)$ in the single-patch group. No patient in the Warden group had late pulmonary vein stenosis. D, The overall incidence of superior vena cava (SVC) obstruction in our series. This occurred in 4 patients $(10 \%)$ in the Warden group, 3 patients $(5 \%)$ in the single-patch group, and 1 patient (4\%) in the 2-patch group. 


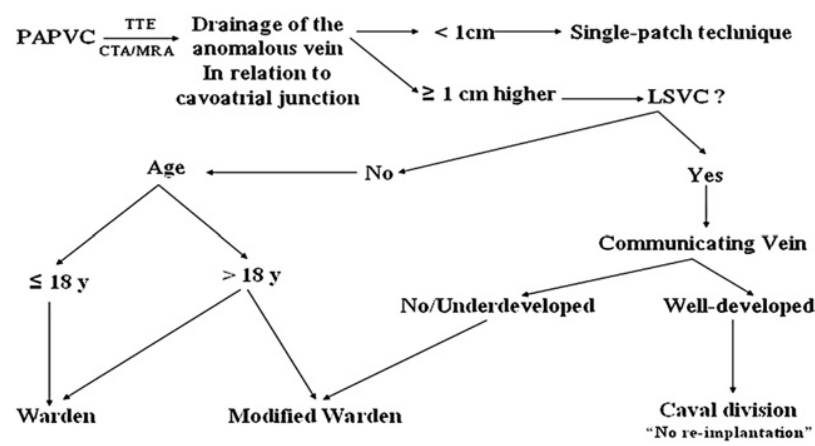

FIGURE 5. Our preferred algorithm for surgical repair of partial anomalous pulmonary venous connection to the right side of the heart. PAPVC, Partial anomalous pulmonary venous connection; $S V C$, superior vena cava; TTE, transthoracic echocardiography; $C T$, computed tomography; $M R A$, magnetic resonance angiography.

pulmonary venous obstruction despite untreated autologous pericardium being the most common patch material used in these patients. This suggests that the adequacy of the patch, rather than the material itself, might be the main factor behind the development of late baffle obstruction. This group of patients require regular follow-up for early detection of SVC or pulmonary venous obstruction. Nicholson and colleagues ${ }^{12}$ reported the use of a lateral caval approach for repair of PAPVC in 66 patients. The mean age at repair was 10.2 years, with a mean follow-up of 4.1 years, and included the use of a 24-hour ambulatory Holter monitor. There were no mortality and no evidence of SVC or pulmonary venous obstruction. All were in sinus rhythm with no evidence of arrhythmias.

Dysrhythmias are known morbidities with these techniques. Sinus node dysfunction can occur with incisions involving the SVC or close to the cavoatrial junction. ${ }^{13}$ Junctional rhythm was identified in 4 after single-patch repair in the series by Iyer and colleagues, ${ }^{7}$ with no other rhythm abnormalities with the 2-patch technique. Rhythm abnormalities were observed in only 2 patients among the 18 patients who underwent cavoatrial incisions in the series by DeLeon and colleagues. ${ }^{14}$ Gaynor and colleagues ${ }^{15}$ did not report any sinus node dysfunction among the 11 patients who underwent Warden procedure. The same was found in the series reported by Stewart and colleagues ${ }^{9}$ of 5 Warden patients. It is thought that the caval division procedure is associated with a lower incidence of sinus node dysfunction. This is because extending the right atrial incision across the cavoatrial junction or near the sinus node is not necessary. We noted a change in the normal sinus rhythm to a junctional rhythm in 5 patients $(21 \%)$ in the 2-patch group, 8 patients $(20 \%)$ in the caval division group, and 7 patients $(12 \%)$ in the single-patch group. At late follow-up, all but 3 patients remain in sinus rhythm. A greater incidence of supraventricular arrhythmias was found in the caval division group $(22 \%)$ than in the 2-patch $(12 \%)$ or single-patch $(8 \%)$ groups. Three patients among our series developed sinus node dysfunction: two in the 2-patch group $(8 \%)$ and one in the caval division group $(2 \%)$. No patient in the single-patch group had sinus node dysfunction. No significant difference was found among the 3 groups regarding the occurrence of dysrhythmias or sinus node dysfunction. This points to the importance of careful placement of the suture line, and adequate design of the patch.

In conclusion, excellent surgical outcomes can be expected when using the single-patch, 2-patch, or caval division technique for repair of PAPVC. The incidence of SVC or pulmonary venous obstruction is low. The 2-patch technique tends to have a greater, although nonsignificant, incidence of pulmonary vein obstruction, sinus node dysfunction, and supraventricular dysrhythmias than the single-patch technique. Adequate sizing of the intra-atrial patch and careful placement of the suture line are mandatory to avoid such morbidities. Regular follow-up is required for early detection of such complications. Figure 5 illustrates our management algorithm for patients with PAPVC.

\section{References}

1. Healey JE Jr. An anatomic survey of anomalous pulmonary veins: their clinical significance. J Thorac Surg. 1952;23:433-44

2. Garduno C, Chew S, Forbess J, Smith PK, Grocott HP. Persistent left superior vena cava and partial anomalous pulmonary venous connection: incidental diagnosis by transesophageal echocardiography during coronary artery bypass surgery. J Am Soc Echocardiogr. 1999;12:682.

3. Trusler GA, Kazenelson G, Freedom RM, Williams WG, Rowe RD. Late results following repair of partial anomalous pulmonary venous connection with sinus venosus atrial septal defect. J Thorac Cardiovasc Surg. 1980;79:776-81.

4. Stewart S, Alexon C, Manning J. Early and late results of repair of partial anomalous pulmonary venous connection to the superior vena cava with a pericardia baffle. Ann Thorac Surg. 1986;41:498-501.

5. Freiedli B, Guerin R, Davignon A, Fouron JC, Stanley P. Surgical treatment of partial anomalous pulmonary venous drainage: a long-term follow-up study. Circulation. 1972;45:159-70.

6. Robicsek F, Daugherty HK, Cook JW, Selle JG. Sinus venosus type of atrial septal defect with partial anomalous pulmonary venous return. J Thorac Cardiovasc Surg. 1979;78:559-62.

7. Iyer AP, Somanrema K, Pathak S, Manjunath PY, Pradhan S, Krishnan S. Comparative study of single- and double-patch techniques for sinus venosus atrial septal defect with partial anomalous pulmonary venous connection. $J$ Thorac Cardiovasc Surg. 2007;133:656-9.

8. Gustafson RA, Warden HE, Murray GF. Partial anomalous pulmonary venous connection to the superior vena cava. Ann Thorac Surg. 1995;60(Suppl):614-7.

9. Stewart RD, Bailliard F, Kelle AM, Backer CL, Young L, Mavroudis C. Evolving surgical strategy for sinus venosus atrial septal defect: effect on sinus node function and late venous obstruction. Ann Thorac Surg. 2007;84:1651-5.

10. Warden HE, Gustafson RA, Tarnay TJ, Neal WA. An alternative method for repair of partial anomalous pulmonary venous connection to the superior vena cava. Ann Thorac Surg. 1984;38:601-5.

11. Shahriari A, Rodefeld MD, Turrentine MW, Brown JW. Caval division technique for sinus venosus atrial septal defect with partial anomalous pulmonary venous connection. Ann Thorac Surg. 2006;81:224-30.

12. Nicholson IA, Chard RB, Nunn GR, Cartmill TB. Transcaval repair of the sinus venosus syndrome. J Thorac Cardiovasc Surg. 2000;119:741-4.

13. Tung KSK, James TN, Effler DB, McCormack J. Injury of the sinus node in openheart operations. J Thorac Cardiovasc Surg. 1967;53:814-29.

14. DeLeon SY, Jenny EF, Ilbawi MN, Husayni TS, Quinones JA, Owe EP, et al. Surgical techniques in partial anomalous pulmonary veins to the superior vena cava. Ann Thorac Surg. 1993;55:1222-6.

15. Gaynor JW, Burch M, Dollery C, Sullivan ID, Deanfield JE, Elliot MJ. Repair of anomalous pulmonary venous connection to the superior vena cava. Ann Thorac Surg. 1995;59:1471-5. 


\section{Discussion}

Dr David Campbell (Aurora, Colo). Before I start, I would like to take the privilege of the floor to say as I was driving here this morning coming down I25 and with the sun just coming up and seeing the beautiful Rockies on the right and the plush green plains and Pike's Peak ahead of me, it just reminded me what a privilege it is for us to live in this country we call the USA.

Having said that, I would like to congratulate you Sameh on a wonderful presentation and thank you very much for your and your co-authors to have provided me with the report well in advance of this program. You have shown excellent results, very good outcomes, no mortality, and very low morbidity. Unfortunately, there is really nothing new here.

I would like to make a few points and perhaps emphasize something that you have already.

First of all, you do have a large number of patients here but the median age was about 34.5 years, and of course when H. E. Warden presented his findings, his median age, I think, was about 12 years of age, so there is a much larger number of adult patients here and perhaps there is more interest in the younger group because in the older patients, the growth potential is probably not as much a concern as it might be in very small children.

Second, the patch material that you talked about I think is very important. Each surgeon has their own feeling about which patch material is important and you really did not emphasize it, but it is really not the patch material that is important here but the adequacy of the baffle and particularly potential for growth. Thus, I think that needs to be re-emphasized. What material you use I think is less important.

Finally, $86 \%$ of your patients had 1 or more of the anomalous veins going to the superior vena cava where only about $32 \%$ of your patients underwent a caval division procedure.

My questions to you are, first, with what you have shown here about the 2-patch technique, is there any place anymore in 2011 for the use of a 2-patch technique?

Dr Said. Thanks, Dr Campbell, for your comments. Back to the first point, I agree with you regarding the mean age of our patients. It might reflect the referral pattern.

Regarding the patch material, with some data talking about the potential for obstruction with autologous pericardium, we tend to find that the bovine pericardium is easy to handle and easy to use. With the age of the patients, most of them were already beyond the growth period so it might be a reasonable option at that time.

Dr Campbell. I would just make one point there though. I can show you other reports where 1 particular material has worked better than something else, so I think the point here to be made is not to get bogged down on what material you are actually using for the patch but to make sure that you have an adequate baffle.

Dr Said. I agree. Regarding the question about the 2-patch technique, in our institution we tend to use the single patch most of the time and for patients who had high insertion of the anomalous veins, we had a low threshold to use the caval division. We had good experience with that, and we did not find too many morbidities associated compared with the 2-patch technique.

Dr Campbell. So am I assuming then you no longer use a 2patch technique at all?

Dr Said. No. I would ask Dr Burkhart, who is my senior author, if he would like to comment on that as well.
Dr Campbell. When would you use a 2-patch technique?

Dr Said. The indication for the 2-patch technique was initially for those who had high insertion of the anomalous veins above the cavoatrial junction. We have used it in a few patients and because of the greater incidence of arrhythmia we found that the caval division is much easier to do with very good results; thus, we tend now more to lean toward the caval division.

Dr Burkhart. If I could comment. Dr Said, nice job with the presentation. Dr Campbell, thank you for your comments.

I think that with this study and with some past experiences with the 2-patch techniques, we have tended to avoid the 2-patch technique. We would use single-patch repair and, if we cannot do it secondary to high vein insertion, we do a Warden. We believe the Warden is a good operation that is teachable, as well as reproducible.

Dr Campbell. Thank you. You have answered my question ...

Dr Burkhart. The discussion brings up another important point. With the advances in robotic heart surgery, there are some reporting using a 2-patch technique for high veins robotically. We have performed the single patch robotically for this operation but would avoid the 2-patch technique for the already mentioned reason.

Dr Campbell. Thanks for the information. That point is taken care of.

The next question I would have of you is exactly when would you use the single-patch technique and when would you use the caval division technique?

Dr Said. If the insertion of the anomalous veins is above the cavoatrial junction by about $1 \mathrm{~cm}$, we tend to use the caval division. Otherwise, we were able to reroute the veins with a single patch without any problems. I think that was the initial recommendation also in some of the reports published about the caval division.

Dr Campbell. I think that I and most of the others that do this would totally agree with you.

My last question is, in your adult group-well, I assume it is adult, but in those patients who underwent the caval division, about one half were standard right atrial appendage to proximal SVC and the other half were some form of, I think, ring Gore-Tex is what you used for your position graft. I would have to ask you is there anyplace anymore for even attempting the standard repair of right atrial appendage to proximal SVC or should all those adult patients or those certainly beyond growth have some form of interposition graft. The other part of that is why the ring Gore-Tex as opposed to some other form of material?

Dr Said. Thank you for the question. For the adult patients, we have noted even sometimes with adequate mobilization of the SVC and the RAA and after cutting all the trabeculations, we had 1 patient who required a return to the operating room for SVC obstruction on the first operative day, and I think there might be a place for an interposition graft in these situations if we think there is still some degree of tension on the anastomosis or a potential for obstruction postoperatively.

With the Gore-Tex ring, we had good experience because of the lower tendency for obstruction or kinking. Also, it is a short piece, and we extrapolated our experience from the Fontan procedure in a similar situation here with SVC obstruction.

Dr Campbell. So do you put all those patients on aspirin or some other antiplatelet drug?

Dr Said. For the adults, we use warfarin for 3 months postoperatively. 
Dr Campbell. Well, thank you very much, and I would like to thank the association for the privilege of discussing this study.

Dr Said. Thanks, Dr Campbell.

Dr Pranava Sinha (Washington, DC). Thank you for your excellent presentation, and I totally agree with the authors and Dr. Campbell that the patch material does not matter when you are operating on older patients, but I would argue that it does matter when you are operating on younger patients. A study I am going to be presenting tomorrow clearly shows that bovine pericardium behaves very differently from pericardium of other sources, and it is only recently that we have bovine pericardium available with the fancy anticalcification treatment. The study period that you mentioned was just plain glutaraldehyde bovine pericardium, and it does make a difference.
Dr Said. Thanks for your comment, and I agree. I think that with the patch material, adequate sizing is important to ensure an unobstructed baffle, but the patch material in some situations could matter. There were 2 patients in our series who developed pulmonary venous obstruction, and, unfortunately, 2 of them had untreated autologous pericardium and required reoperation for that reason. We did not have any problem with the use of bovine pericardium until now, and it tends to create an unobstructed pathway.

Dr Sinha. It is not just pulmonary venous obstruction. You also have 3 patients with SVC obstruction with the single-patch technique. Either way, the patch goes, it is going to pinch 1 of the pathways.

Dr Said. Thank you. 